\title{
Rationale and design of the Hunting for the off-target propertles of Ticagrelor on Endothelial function and other Circulating biomarkers in Humans (HI-TECH) trial
}

\author{
Sara Ariotti, MD, ${ }^{a}$ Maarten van Leeuwen, MD, PhD, ${ }^{\mathbf{b}}$ Salvatore Brugaletta, MD, PhD, ${ }^{\mathrm{c}}$ Sergio Leonardi, $\mathrm{MD}, \mathrm{PhD},{ }^{\mathrm{d}}$ \\ Kristiaan Martijn Akkerhuis, MD, PhD, ${ }^{\mathrm{e}}$ Emrush Rexhaj, MD, PhD, ${ }^{\mathrm{a}}$ Gladys Janssens, MD, ${ }^{\mathrm{b}}$ Luis Ortega-Paz, MD, ${ }^{\mathrm{c}}$ \\ Diego Rizzotti, MSc, ${ }^{\mathrm{d}}$ Jan C. van den Berge, MD, ${ }^{\mathrm{e}}$ Dierik Heg, PhD, ${ }^{\mathrm{f}}$ Gloria Francolini, CLS, ${ }^{\mathrm{g}}$ Stephan Windecker, MD, ${ }^{\text {a }}$ \\ and Marco Valgimigli, MD, PhD ${ }^{\text {a }}$, The HI-TECH Investigators Bern, Switzerland; Amsterdam, Rotterdam, the \\ Netberlands; Barcelona, Spain; Pavia, and Brescia, Italy
}

Background Among the 3 approved oral P2 $_{12}$ inhibitors for the treatment for patients with acute coronary syndrome (ACS), ticagrelor, but not prasugrel or clopidogrel, has been associated with off-target properties, such as improved endothelial-dependent vasomotion and increased adenosine plasma levels.

Methods The HI-TECH study (NCT02587260) is a multinational, randomized, open-label, crossover study with a Latin squares design, conducted at 5 European sites, in which patients free from recurrent ischemic or bleeding events $\geq 30$ days after a qualifying ACS were allocated to sequentially receive a $30 \pm 5$-day treatment with prasugrel, clopidogrel, and ticagrelor in random order. The primary objective was to evaluate whether ticagrelor, at treatment steady state (ie, after $30 \pm 5$ days of drug administration), as compared with both clopidogrel and prasugrel, is associated with an improved endothelial function, assessed with peripheral arterial tonometry. Thirty-six patients undergoing evaluable endothelial function assessment for each of the assigned $\mathrm{P} 2 \mathrm{Y}_{12}$ inhibitor were needed to provide $90 \%$ power to detect a $10 \%$ relative change of the reactive hyperemia index in the ticagrelor group.

Conclusion The HI-TECH study is the first randomized, crossover study aiming to ascertain whether ticagrelor, when administered at approved regimen in post-ACS patients, improves endothelial function as compared with both clopidogrel and prasugrel.

\section{Background}

Oral $\mathrm{P}_{2} \mathrm{Y}_{12}$ inhibitors are key secondary prevention medications after coronary stent implantation or acute coronary syndromes (ACSs). Ticagrelor, unlike other oral $\mathrm{P}^{2} \mathrm{Y}_{12}$ inhibitors, has been associated with off-target effects, such as an improvement in endothelial function assessed by peripheral arterial tonometry, as recently reported in a nonrandomized trial. ${ }^{1}$ Moreover, in a recent

From the ${ }^{a}$ Bern University Hospital, Bern, Switzerland, ${ }^{b}$ VU University Medical Center, Amsterdam, the Netherlands, ${ }^{C}$ Cardiovascular Clinic Institute, Hospital Clinic, University of Barcelona, IDIBAPS, Barcelona, Spain, ${ }^{d}$ Fondazione IRCCS Policlinico S. Matteo, Pavia, Italy, ${ }^{e}$ Erasmus University Medical Center, Rotterdam, the Netherlands, ${ }^{f}$ Clinical Trials Unit and Institute of Social \& Preventive Medicine, University of Bern, Bern, Switzerland, and g/stituti Clinici Scientifici Maugeri-IRCCS Lumezzane, Brescia, Italy. NCT02587260

Submitted December 4, 2016; accepted March 25, 2017.

Reprint requests: Marco Valgimigli, MD, PhD, Department of Cardiology, Bern University Hospital, SH1 307, Freiburgstrasse 4, 3010 Bern, Switzerland.

E-mail: marco.valgimigli@insel.ch randomized trial of 60 ACS patients, 30-day ticagrelor administration was shown to increase reactive hyperemia index (RHI) by $100 \%$ as compared with baseline measurement, and this improvement was correlated with adenosine plasma levels (APLs). ${ }^{2}$ A recent observational study also reported no change in endothelial function at 2 and 5 days after treatment discontinuation with ticagrelor, which was interpreted as evidence that the treatment effect on endothelial function does not immediately cease after treatment cessation. ${ }^{3}$

Endothelial dysfunction is a systemic condition mainly characterized by an imbalance between endotheliumderived relaxing factors (ie, nitric oxide $[\mathrm{NO}])^{4}$ and endothelium-derived contracting factors (ie, endothelin), ${ }^{5}$ clinically correlated with most cardiovascular risk factors. ${ }^{6}$ Endothelial dysfunction appears to precede the clinical manifestation of atherosclerotic disorders and predicts clinical outcome. ${ }^{7}$ Thus, it can be considered a barometer of the total risk burden. ${ }^{8-10}$

The "ticagrelor-related pleiotropic effects" are possibly mediated by the inhibition of adenosine uptake into 
Table. Inclusion and exclusion criteria

Inclusion criteria

1. Age $>18 \mathrm{y}$

2. ACS (including STEMI or NSTEMI) at least $30 \mathrm{~d}$ before randomization

3. Ongoing treatment with DAPT since at least $30 \mathrm{~d}$, consisting of ASA 75-160 mg daily and 1 of the 3 available P2Y 12 inhibitors (ticagrelor, prasugrel, or clopidogrel)

4. No bleeding events (defined as BARC type 2 or greater) or ischemic recurrences in the period between the ACS and the study randomization

Exclusion criteria

1. Administration of fibrinolytics or glycoprotein IIb/IIIa inhibitors in the previous $30 \mathrm{~d}$

2. Major surgery within $30 \mathrm{~d}$ or any planned surgical or percutaneous intervention

3. Active bleeding or previous clinically relevant bleeding in the last 6 mo

4. Previous TIA or stroke in the last 6 mo

5. Previous intracranial bleeding

6. Thrombocytopenia

7. Ongoing anticoagulant therapy or clinical indication to start with anticoagulant agents

8. Vasculitis or any known immunological disorder

9. Severe hepatic failure

10. Uncontrolled hypertension (systolic or diastolic arterial pressure $>180 \mathrm{mmHg}$ or $120 \mathrm{mmHg}$, respectively, despite medical therapy)

11. Known intolerance to aspirin, clopidogrel, prasugrel, or ticagrelor

12. Limited life expectancy (ie, neoplasms)

13. Inability to obtain the informed consent

14. Pregnancy

Abbreviations: STEMI, ST-segment elevation myocardial infarction; NSTEMI, non-ST-segment elevation myocardial infarction; ASA, aspirin; BARC, Bleeding Academic Research Consortium; TIA, transient ischemic attack.

erythrocytes and subsequent increase in APLs, as recently reported. ${ }^{2,11,12}$

Adenosine is released in the plasma by endothelial cells and myocytes during ischemia, hypoxia, or oxidative stress, and quickly taken up by red blood cells through a facilitated diffusion transport system (sodium-independent equilibrative nucleoside transporters 1 and 2; sodium-dependent concentrative nucleoside transporters 1 and 2) or converted into inosine by adenosine deaminase activity. Ticagrelor increases APL mainly through inhibition of equilibrative nucleoside transporter 1. ${ }^{11}$ After binding to 4 different purinergic receptors $\left(A_{1}\right.$, $A_{2 A}, A_{2 B}, A_{3}$ ), an increase in APL may determine (1) vasodilation, (2) reduction in ischemia/reperfusion injury and electrical conduction, (3) increase of platelet inhibition, (4) decrease of glomerular filtration rate, and (5) rise of dyspnea incidence. Finally, ticagrelor induces adenosine triphosphate release from human red blood cells in a dose-dependent manner, ${ }^{13}$ which may contribute to increasing APL. It remains, however, unclear whether the off-target properties of ticagrelor, which were discovered and characterized in animals or ex vivo models, ${ }^{14,15}$ contribute to its clinical effects in humans at currently approved regimen.

\section{Methods}

Study design and population

The HI-TECH study (ClinicalTrial.gov NCT02587260) is a multinational, randomized, open-label, crossover study with a Latin squares design conducted at 5 European sites and including 54 patients, aiming to assess whether ticagrelor, as compared with both clopidogrel and prasugrel, improves endothelial function. Eligible patients were older than 18 years treated for an ACS, including ST-segment elevation myocardial infarction, non-ST-segment elevation myocardial infarction, or unstable angina, at least 30 days before randomization, and receiving dual antiplatelet therapy (DAPT) since at least 30 days before randomization. Patients were free from bleeding events (defined as Bleeding Academic Research Consortium classification ${ }^{16}$ type 2 or greater) or ischemic recurrences (unstable angina or myocardial infarction, which required a repeated revascularization) since the index event. Patients with transient ischemic attack or stroke in the previous 6 months, those who received fibrinolytic therapy or glycoprotein IIb/IIIa inhibitors in the previous 30 days; those with indication for oral anticoagulant therapy, or with vasculitis, immunological disorders, thrombocytopenia, severe hepatic 
Figure 1

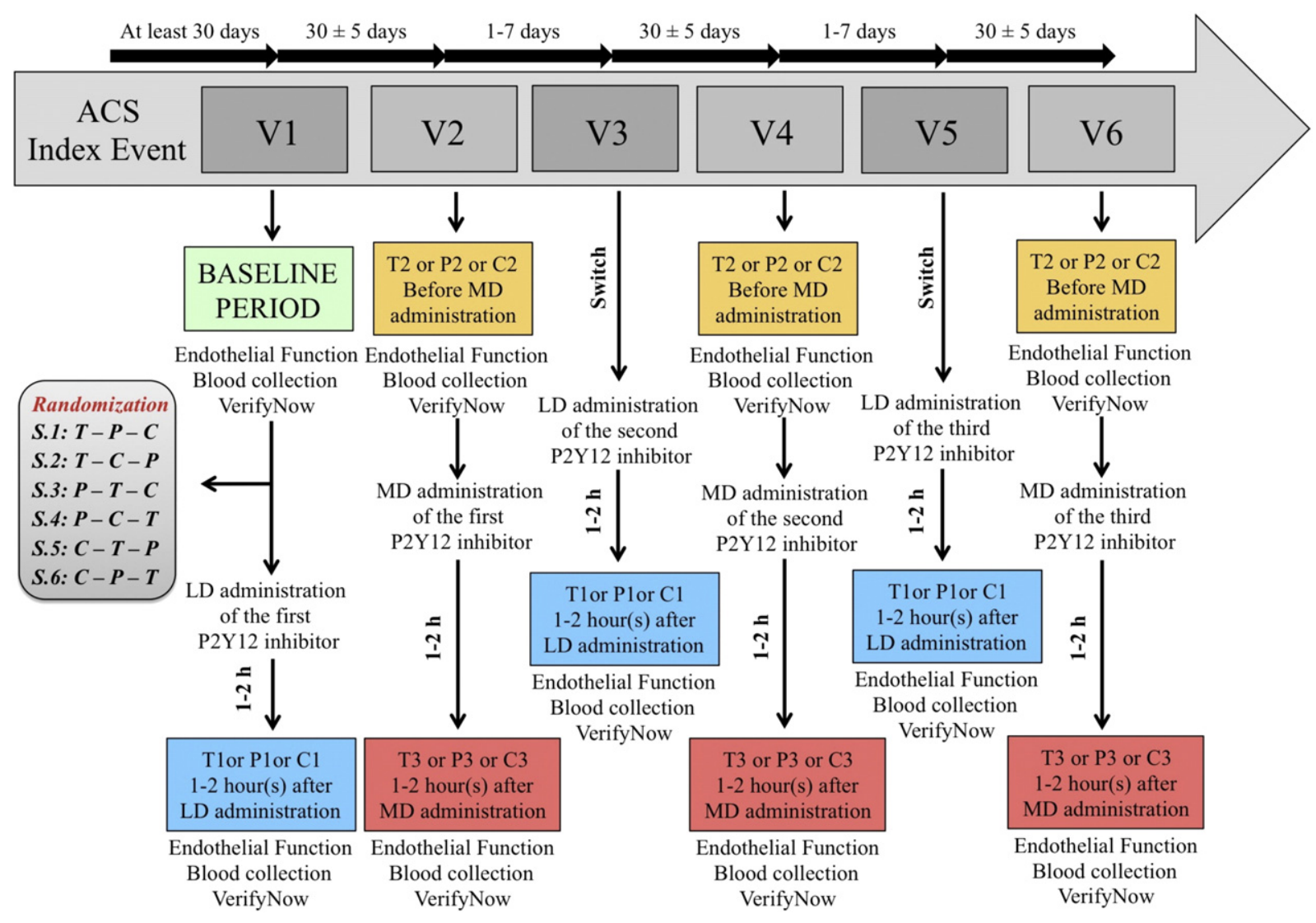

Study design. Study flowchart depicting study visits with the respective time intervals (black arrows on top) and all procedures performed at each time point. Endothelial function evaluation is performed using EndoPAT in all sites, whereas FMD was performed only for patients recruited in Bern. Blood was collected for dosage of circulating biomarkers. VerifyNow measurements, using both $\mathrm{P} 2 \mathrm{Y}_{12}$ and ASA assays, were obtained to assess platelet reactivity. $\mathrm{V} 1$ indicates visit $1 ; \mathrm{V} 2$, visit $2 ; \mathrm{V} 3$, visit $3 ; \mathrm{V} 4$, visit 4 ; $\mathrm{V} 5$, visit 5 ; V6, visit 6; MD, maintenance dose; $\mathrm{T1}$, 1-2 hours after ticagrelor LD administration; T2, before ticagrelor MD administration; T3, 1-2 hours after ticagrelor MD administration; P1, 1-2 hours after prasugrel LD administration; P2, before prasugrel MD administration; P3, 1-2 hours after prasugrel MD administration; C1, 1-2 hours after clopidogrel LD administration; C2, before clopidogrel MD administration; C3, 1-2 hours after clopidogrel MD administration; $S$, sequence; $\mathrm{P}$, prasugrel; T, ticagrelor; C, clopidogrel.

failure, uncontrolled hypertension (systolic or diastolic pressure $>180$ or $120 \mathrm{mmHg}$, respectively, despite medical therapy), or known intolerance to aspirin, clopidogrel, prasugrel, or ticagrelor; and those with limited life expectancy (eg, neoplasm) as well as patients who underwent major surgery within 30 days before randomization or with any planned surgical or percutaneous intervention were excluded (Table).

A Latin square design was used to have a uniform crossover design in that each treatment occurred only once within each sequence and once within each period. Moreover, each treatment preceded every other treatment the same number of times (twice), and consequently, our design was balanced with respect to first-order carryover effects (balanced Latin square design). The crossover study design was chosen so to have an "in-within" control of the effect of each $\mathrm{P}_{2} \mathrm{Y}_{12}$ inhibitor on endothelial function (in the same patient) as opposed to an "in-between" control (different patients). Supplementary Table I displays the 6 randomized treatment sequences. Adherence to study treatment was assessed by electronic Medication Event Monitoring System, pill count, and patient interview (see Supplementary Appendix).

\section{Follow-up visits}

The study included 6 follow-up visits, counting a total of 10 study intervals: baseline (B), 1-2 hours after loading dose (LD) intake of each $\mathrm{P}_{2} \mathrm{Y}_{12}$ inhibitor (C1, P1, T1), and before (C2, P2, T2) and 1-2 hours after MD intake of each $\mathrm{P}_{12}$ inhibitor (C3, P3, T3) (Figure 1). The LD of the first randomized $\mathrm{P}_{2} \mathrm{Y}_{12}$ inhibitor was administered at visit 1 , 


\section{Figure 2}
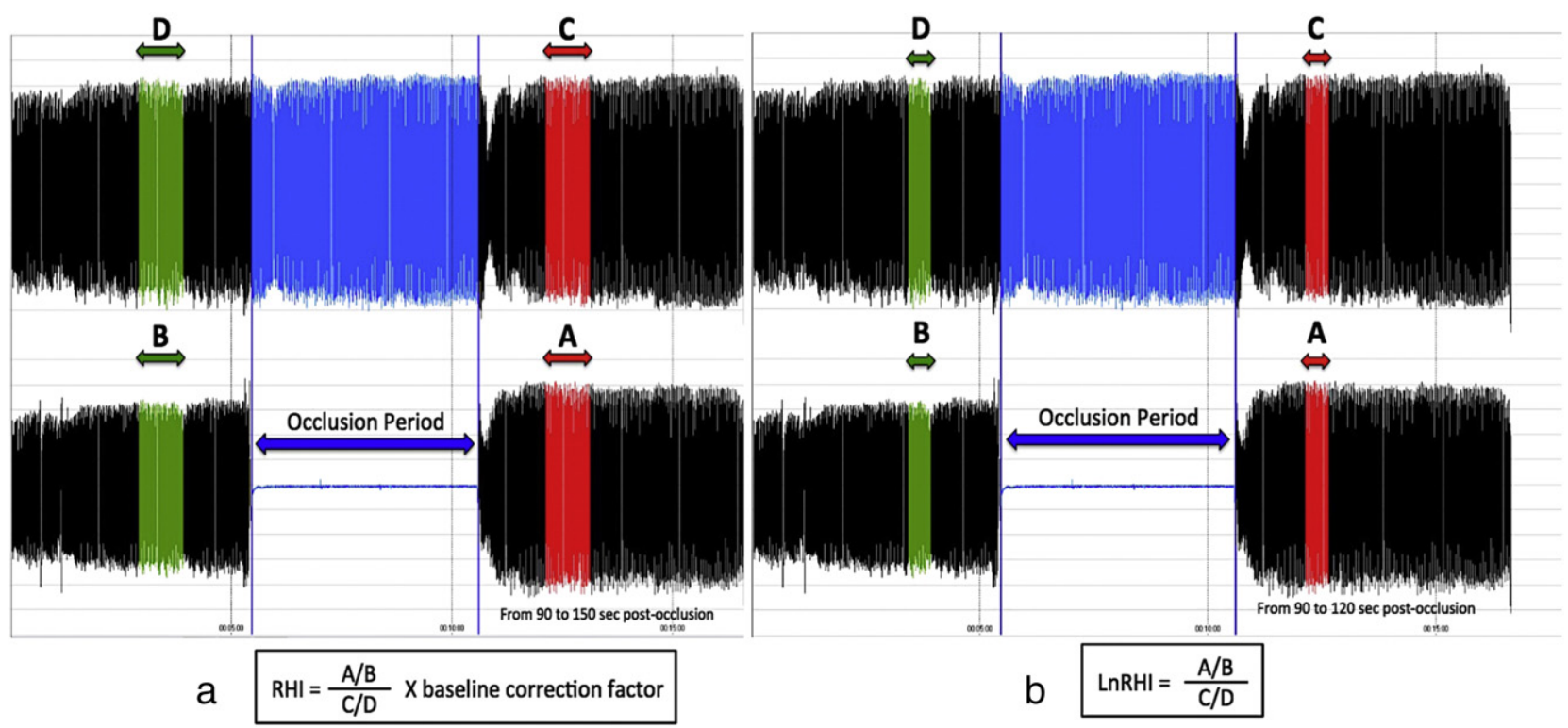

EndoPAT measurement. A, RHI measurement after EndoPAT assessment. A = mean PAT amplitude between 90 and $150 \mathrm{~s}$ after occlusion of the test arm; $B=$ mean PAT amplitude from the baseline period on the test arm; $C=$ mean PAT amplitude between 90 and $150 \mathrm{~s}$ after occlusion of the control arm; $D=$ mean PAT amplitude from the baseline period of the control arm. $\mathbf{B}$, LnRHI measurement after EndoPAT assessment. $A=$ mean PAT amplitude between 90 and $120 \mathrm{~s}$ after occlusion of the test arm; $B=$ mean PAT amplitude from the baseline period on the test arm; $C=$ mean PAT amplitude between 90 and $120 \mathrm{~s}$ after occlusion of the control arm; D = mean PAT amplitude from the baseline period of the control arm.

after baseline assessment, followed by post-LD measurements 1-2 hours thereafter (Figure 1). Pre- and post-MD evaluations of the ongoing $\mathrm{P}_{2} \mathrm{Y}_{12}$ inhibitor were performed after $30 \pm 5$ days of treatment at visit 2 (V2). The administration of the second randomized $\mathrm{P} 2 \mathrm{Y}_{12}$ inhibitor at visit 3 occurred 1 to 7 days after visit 2 , and the related measurements were collected 1-2 hours after LD administration (Figure 1). An identical scheme was then followed for the second and third randomized $\mathrm{P}_{2} \mathrm{Y}_{12}$ inhibitor (Figure 1). No washout time was allowed among the 3 oral $\mathrm{P}_{2} \mathrm{Y}_{12}$ inhibitors because of ethics considerations. At each follow-up, patients were requested to fast for at least 2 hours before the visit; caffeine-containing beverages were not permitted at any time the day of the visit. Patients were reminded 1-2 days before each study visit about these dietary restrictions.

\section{Finger plethysmography}

Pulse amplitude tonometry (PAT) is an operator-independent, Food and Drug Administration-approved method to measure the endothelium-dependent dilation in response to reactive hyperemia. ${ }^{10}$ The PAT device records digital pulse wave amplitude using fingertip plethysmography (EndoPAT; Itamar Medical Ltd, Caesarea, Israel) and quantifies the endothelium-mediated changes in vascular tone, elicited by a 5-minute occlusion of the brachial artery. A postocclusion-to-preocclusion ratio (Figure 2) is calculated by the EndoPAT software and expressed as RHI (Figure 2A) or its natural logarithm variant (LnRHI; Figure 2B). These values are normalized to measurements from the contralateral arm, which serves as control for nonendothelial dependent systemic effects. An RHI value less than 1.67 or an LnRHI value less than 0.51 denotes an endothelial dysfunction. ${ }^{8}$ EndoPAT was found to be well correlated with coronary endothelial function, evaluated by quantitative coronary angiography after injection of acetylcholine, ${ }^{8}$ as well as with the conventional cardiovascular risk factors. ${ }^{17,18}$ EndoPAT measurement was able to identify patients with early coronary atherosclerosis ${ }^{8}$ and predicts late cardiovascular adverse events. ${ }^{10}$ EndoPAT reliability was tested in healthy adults and adolescents, and it was shown to be highly reproducible across intervals of 1 day ${ }^{19}$ and 1 week or longer. $^{20-22}$ Description of technical procedure is reported in Supplementary Appendix.

Methodological advantages of this technology are as follows: (1) simultaneous recording from both arms with an intrasubject control, (2) live assessment of occlusion and provocation quality to avoid incomplete occlusion during the examination, (3) large dynamic range of measurements due to the finger ability to vary local vascular tone, and (4) operator independency as all analyses are automated. Limitations to this assessment include the following: (1) the assessment of endothelial function at a microcirculation level instead of at medium 
Figure 3

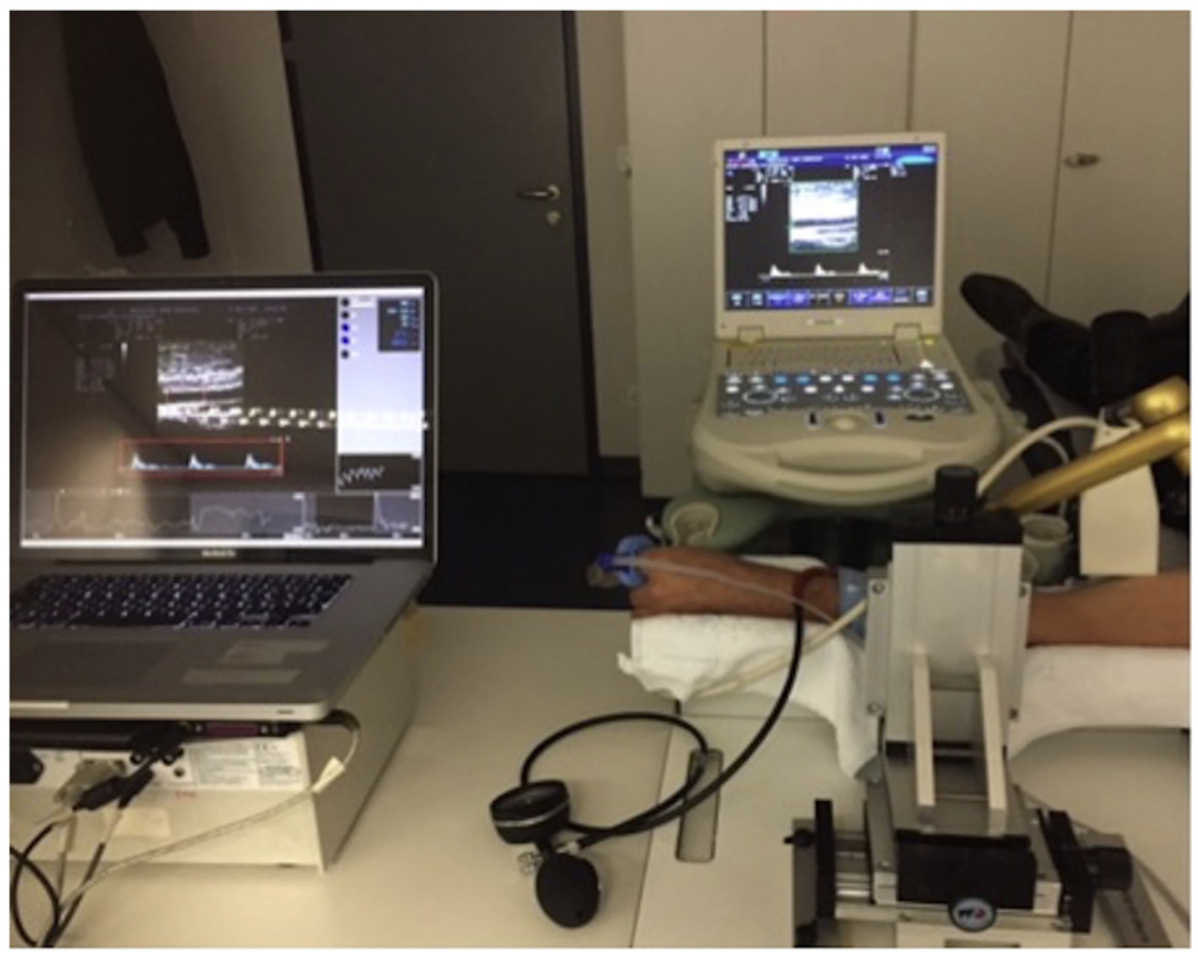

Assessment of FMD and EndoPAT at Bern University Hospital. The picture shows the concomitant acquisition of FMD and EndoPAT measurements at Bern University Hospital.

to large size vessel such as the brachial artery, as assessed via standard flow-mediated dilatation; (2) the use of a fixed time frame during the hyperemic response to calculate the EndoScore, whereas the maximal hyperemic response can occur with different delays for each patient, especially in older subjects ${ }^{23}$; (3) the influence of autonomous nervous system or the temperature study room on finger vascular tone; and (4) limited knowledge about the effect of circadian variation on RHI result.

\section{Flow-mediated dilation}

Flow-mediated dilation (FMD) of brachial artery is a noninvasive technique widely used to assess endothelial function. The FMD result is reported as a percentage change between the peak diameter in response to reactive hyperemia, after 5 minutes of artery occlusion, and the baseline diameter using the following equation:

$$
\begin{aligned}
& \text { FMD }(\%)= \\
& \quad(\text { Peak diameter-Baseline diameter }) / \text { Baseline diameter }{ }^{*} 100 .
\end{aligned}
$$

Moreover, the FMD evaluation after nitrate administration is able to differentiate the endothelium-independent vasodilation component due to alterations in smooth and not endothelial cell function, improving the endothelial function assessment. However, although the principle seems simple and many laboratories showed robust test-retest reliability for FMD under standardized conditions, ${ }^{2-26}$ appropriate high-resolution ultrasound equipment and highly skilled personnel are essential for obtaining an accurate and reliable measurement. $^{18,20,27}$ Ghiadoni et al $^{27}$ evaluated the FMD twice in the same day and subsequently after 30 days after a dedicated training program in the setting of a multicenter evaluation of 135 healthy volunteers. Values of FMD did not differ over time and showed an intrasession coefficient of variation ranging from $7.6 \%$ to $11.9 \%$ and an intersession coefficient of variation ranging from $11.6 \%$ to $16.1 \%$ across centers. No data have been, however, provided in patients with coronary artery disease who may have higher intrasession and intersession variability. Furthermore, it remains challenging to standardize FMD protocol and technical expertise across different sites. Therefore, we evaluated the endothelial function using the FMD jointly to the EndoPAT technology (Figure 3), only in one study center-Bern University Hospital, Switzerland, with large prior experience with this methodology. ${ }^{28-31}$ Description of technical procedure is reported in Supplementary Appendix.

\section{Circulating biomarkers}

Plasma levels of various circulating biomarkers have been associated with the presence of endothelial 
dysfunction. Endothelin-1 is a 21-amino-acid peptide synthesized from a larger preproET-1 precursor that elicits its vasoconstrictor properties binding 2 G-proteincoupled receptors, $\mathrm{ET}_{\mathrm{A}}$ and $\mathrm{ET}_{\mathrm{B}}$, located on vascular smooth muscle cells, fibroblasts, and endothelial cells ( $\mathrm{ET}_{\mathrm{B}}$ only). Endothelin-1 induces endothelial dysfunction reducing NO bioavailability through 2 different ways: (1) decreasing its production via caveolin-1-mediated inhibition of eNOS activity and (2) increasing its degradation via formation of oxygen radicals. ${ }^{32}$ Accordingly, high levels of ET-1 may represent an indirect index of endothelial dysfunction.

Asymmetric dimethylarginine (ADMA) is an analogue of L-arginine found in human circulation and represents a naturally occurring endogenous inhibitor of NO synthase. ${ }^{33}$ Reducing NO production, ADMA could thus lead to endothelial dysfunction and cardiovascular events. Elevated plasma ADMA concentration has been identified as an independent risk factor for progression of atherosclerosis, cardiovascular death, and all-cause mortality. ${ }^{3436}$

von Willebrand factor (vWF) is a multimeric glycoprotein synthesized exclusively in endothelial cells and megakaryocytes and released when endothelial cells are damaged. Thus, high level of circulating vWF may reflect endothelium damage or endothelial dysfunction. The close association between vWF and thrombogenensis or atherogenesis also suggests that high vWF levels may be a useful indirect indicator of atherosclerosis and/or thrombosis. ${ }^{37}$

Blood collection was to be performed after EndoPAT assessment at each time point, using the control arm as sampling site for the quantification of ADMA, vWF antigen, and ET-1. To assess the relationship between residual platelet reactivity or percentage inhibition and effect of $\mathrm{P}_{2} \mathrm{Y}_{12}$ oral blocker on endothelial function, a platelet function testing was also to be carried out using the VerifyNow (Accumetrics - Accriva diagnostics - United States) system using both $\mathrm{P}_{2} \mathrm{Y}_{12}$ and aspirin assays.

Markers of thrombin activity such as prothrombin fragment $1+2$, fibrinopeptide $\mathrm{A}$, and thrombin-antithrombin complex (TAT) were also measured for exploratory purpose. All circulating biomarkers reported above were predefined and will be analyzed by a centralized laboratory (Istituti Clinici Scientifici Maugeri, IRCCS Lumezzane, Brescia, Italy). C-reactive protein will be measured with an immunoturbidimetric assay, whereas all the other biomarkers will be measured using commercially available enzyme-linked immunosorbent assay tests (Supplementary Table II), including the vWF antigen, for which the enzyme-linked immunosorbent assay test is a well-validated method of measurement. ${ }^{38-40}$ Finally, to provide mechanistic data on how ticagrelor may be associated with improved endothelial function, adenosine plasma concentration, ticagrelor and its metabolite AR-C124910XX will be dosed in 2 different reference laboratories (Q\&Q Labs AB, Bio VentureHub, Mölndal, Sweden, and Bioanalytical Covance Laboratory, Indianapolis, IN, respectively). An overview of all investigated biomarkers with the corresponding collection time points is shown in Supplementary Table I. Protocols of sample collection, centrifugation, and storage were standardized in all sites (preanalytical standardization) and are reported in Supplementary Table IV. When immediately frozen at $-20^{\circ} \mathrm{C}$ (as stored in Amsterdam, Barcelona, Pavia, and Bern), or $-80^{\circ} \mathrm{C}$ (as stored in Rotterdam), after centrifugation and never thawed, all assessed biomarkers are known to be stable in vitro for a long time (at least few years). The only exception is represented by adenosine, which is immediately degraded after blood collection with a half-life of a few seconds. To improve adenosine stability and allow its subsequent dosage, a stopping solution was immediately added to blood tube during sampling.

\section{Collection of adenosine samples}

Adenosine plasma samples were collected as described by Bonello et al. ${ }^{12}$ Venous blood $(2.6 \mathrm{~mL})$ was withdrawn under vacuum together with a STOP solution previously placed in S-Monovette $2.6 \mathrm{~mL}$, K3 EDTA (Sarstedt, Nümbrecht, Germany). This method allows blood sample to be mixed rapidly with $4 \mathrm{~mL}$ of STOP solution, which prevents adenosine degradation and uptake. The STOP solution was composed of NBMPR $0.1 \mathrm{mml} / \mathrm{L}$, dipyridamole $0.04 \mathrm{mmol} / \mathrm{L}$, AMPCP $0.22 \mathrm{mmol} / \mathrm{L}$, ethylene-diaminetetraacetic acid disodium salt dehydrate $15 \mathrm{mmol} / \mathrm{L}$, 5-iodotubericidin $0.1 \mathrm{mmol} / \mathrm{L}$, and erythro-9-(2hydroxy-3-nonyl)-adenine $0.1 \mathrm{mmol} / \mathrm{L}$ dissolved in phosphatebuffered saline, $\mathrm{pH} 7.4,0.01 \mathrm{mmol} / \mathrm{L}$ (Supplementary Table V). The sample with the STOP solution was centrifuged at $1640 \mathrm{~g}$ for 10 minutes at room temperature. The plasma supernatant was then transferred in a dedicated microtube and then stored at $-20^{\circ} \mathrm{C}$ or, preferably, $-80^{\circ} \mathrm{C}$.

\section{Randomization}

Allocation of study treatment was performed via a Web-based interactive randomization system available at https://trials.advicepharma.com/hitech. Randomization was achieved with computer-generated random sequence with a random block size (from $\mathrm{X}$ to $\mathrm{Y}$ ) stratified according to the clinical site and the presence of diabetes mellitus.

\section{Treatment protocol and follow-up}

All patients received DAPT for the entire duration of the study, which included aspirin (75-160 mg/d orally) and, in random order, clopidogrel (600 mg orally as LD followed by $75 \mathrm{mg} / \mathrm{d}$ ) for $30 \pm 5$ days, prasugrel ( $60 \mathrm{mg}$ orally as LD followed by $10 \mathrm{mg} / \mathrm{d}$, or $5 \mathrm{mg} / \mathrm{d}$ if age $\geq 75$ years and/or weight $\leq 60 \mathrm{~kg}$ ) for $30 \pm 5$ days, and ticagrelor $(180 \mathrm{mg}$ orally as LD followed by $90 \mathrm{mg}$ twice a day) for $30 \pm 5$ days. Follow-up visit schedule is shown in Figure 1.

\section{Study end points}

The primary objective of the study is RHI at treatment steady state (ie, after $30 \pm 5$ days of treatment), evaluated with EndoPAT system and assessed 1-2 hours after intake 
of the daily (for clopidogrel or prasugrel) or morning (for ticagrelor) maintenance dose of each investigated $\mathrm{P}_{2} \mathrm{Y}_{12}$ inhibitor. The primary end point consists of 2 main comparisons: ticagrelor vs prasugrel difference in RHI, and ticagrelor vs clopidogrel difference in RHI.

The secondary objectives include RHI 1-2 hours after $\mathrm{P}_{2} \mathrm{Y}_{12}$ inhibitor LD or prior MD administration and other biomarkers of endothelial function. Each of these secondary end points encompasses 2 main comparisons: ticagrelor vs prasugrel difference, and ticagrelor vs clopidogrel difference. The comparison between prasugrel and clopidogrel in terms of RHI and circulating biomarkers will be also reported for exploratory purposes.

\section{Statistical considerations}

The null hypothesis (HO) of this study is that the primary end point (difference in RHI at treatment steady state) does not differ during ticagrelor treatment as compared with prasugrel or clopidogrel. The alternative hypothesis (H1) is that the primary end point differs after ticagrelor as compared with prasugrel or clopidogrel. Sample size calculation was based on repeated 2-way analysis of variance (ANOVA), ${ }^{20}$ setting mean RHI at 1.8 with a within-subjects SD of 0.31 . Hence, 36 patients completing all sequences (ie, 6 patients/sequence) provides $90 \%$ power to detect a $10 \%$ RHI relative change in ticagrelor group with a 2-sided $\alpha$ level at 5\%. To account for dropouts as well as incomplete data assessments, the final sample size was increased up to $\geq 50$ patients.

The primary end point will be analyzed using repea-tedmeasures on1-factorial ANOVA (ANOVA with 3 levels as treatment factor to account for each of the 3 tested $\mathrm{P}_{2} \mathrm{Y}_{12}$ inhibitor). Correction for possible intragroup correlation will be done by the Greenhouse-Geisser method. The ANOVA will yield the differences between the 2 main comparisons, ticagrelor RHI vs prasugrel RHI and ticagrelor RHI vs clopidogrel RHI. To assess the primary end point, the significance of these 2 main comparisons will be combined using the Hochberg-Benjamini method $^{41}$ as follows: the Ho of randomized treatment equivalence comparing the response in RHI after ticagrelor vs prasugrel administration and the response in $\mathrm{RHI}$ after ticagrelor vs clopidogrel administration is rejected if significance is achieved for both main comparisons at a 2 sided $\alpha$ level of .05 (ie, the difference in RHI ticagrelor vs prasugrel is supported with $P$ value $<.05$ and the difference in RHI ticagrelor vs clopidogrel is supported with $P$ value $<.05$ ), or for one comparison at a 2-sided $\alpha$ level of .025 (ie, the difference in RHI ticagrelor vs prasugrel is supported with $P$ value $<.025$ or the difference in RHI ticagrelor vs clopidogrel is supported with $P$ value $<.025$ ).

Each of the secondary end points will be analyzed using the same predefined statistical assumptions, combining the $P$ values of the 2 main comparisons. The HO will be rejected if either comparison yields a $P$ value $<.025$ or when both comparisons yield $P$ values $<.05$. See Supplementary appendix for further explanation and for examples regarding the application of the BenjaminiHochberg in our experimental setting (Supplementary Table VI).

The third comparison (ie, the difference in response between prasugrel and clopidogrel) will be reported as explorative unpowered end point.

The SWAP-2 study showed that switching over to prasugrel from previous treatment with ticagrelor results in a progressive decline in PLT inhibition (leading to a clear rebound effect if no LD is given) as compared with the continuation of treatment with ticagrelor. Hence, it may be assumed that any measurement performed during the early phase of any switch from one to another P2Y12 inhibitor may be confounded (ie, it may also at least partially reflect prior exposure to the earlier P2Y12 inhibitor). Based on the timing of blood sampling in this study, one may assume this carryover effect to disappear from after 48 hours and up to 7 days. This was the rational for setting the primary EP measure remotely, that is, at 30 days after each crossover. Nevertheless, methodologies that account for any possible carryover effect, such as analysis of covariance and stratified analysis based on the randomized sequence as well as nonrandomized type of $\mathrm{P}_{12} \mathrm{Y}_{12}$ inhibitor before randomization, will be applied for multiple sensitivity analyses for all primary and secondary end points. It is also prespecified that for these sensitivity analyses, clopidogrel and prasugrel will be handled separately as well as lumped together in the thienopyridines group.

Stratified analysis of the primary end point will be also carried out according to sex, age, presence of diabetes, hypertension, active smoking, dyslipidemia, body mass index, study site, number of vessels diseased, type of ACS at presentation, randomization sequence, and prerandomization $\mathrm{P}_{2} \mathrm{Y}_{12}$ inhibitor intake.

\section{Predefined subanalyses and substudies}

Prespecified subanalyses and substudies include, but are not limited to, the evaluation of adenosine plasma concentration, adherence to study drugs, and effect of age or concomitant drugs on primary or secondary end point measures of endothelial function and FMD results.

\section{Study organization}

The HI-TECH study was conducted at 5 investigative sites in 4 European countries, including Switzerland, Italy, Spain, and the Netherlands. The final study protocol and informed consent have been reviewed and approved by the ethics boards/institutional review boards and corresponding health authorities for all participant study sites/countries. The study is an investigator-driven clinical trial partially supported by an unrestricted research grant from AstraZeneca. The authors are solely responsible for the design and conduct of this study; all study analyses; and drafting and editing of the manuscript. Data are being coordinated and analyzed by an 
academic Clinical Trial Unit located in Bern, Switzerland. The trial registration number is NCT02587260 available at https://register.clinicaltrials.gov/prs/app/action/ LoginUser?uid=U0002SC5\&ts=167\&cx=-f91kzu. Independent study monitoring was performed by AdvicePharma (Milan, Italy). The Electronic Data Capture was designed by the investigators and Web-implemented by AdvicePharma.

\section{Conclusions}

The HI-TECH study is the first randomized, crossover study aiming to ascertain whether ticagrelor, when administered at approved regimen in post-ACS patients, improves endothelial function as compared with both clopidogrel and prasugrel. The first patient was randomized on December 2015 and the last on October 2016 with a total of 54 patients. The last follow-up visit was performed on February 2017 and the final results are expected in the third quarter of 2017.

\section{Disclosures}

Salvatore Brugaletta received lectures fees from AstraZeneca, Abbott, and Boston, and has received institutional research grant from AstraZeneca. Sergio Leonardi received personal fees from AstraZeneca, The Medicine Company, Merck, Daiichi Sankyo, and Ely Lilly, and institutional research grants from AstraZeneca and Daiichi Sankyo. K. Stephan Windecker received research contracts to the institution from Abbott, Biotronik, Boston Scientific, Medtronic, Edwards Lifesciences, and St Jude. Marco Valgimigli has received speaker's fees from AstraZeneca, Biosensor, and Terumo, and has received institutional research grants from AstraZeneca, The Medicines Company, and Terumo. Other authors have nothing to declare.

\section{Contributorship}

M.V. designed the study and obtained funding. M.V. and S.A. contributed to protocol development. M.V. and S.A. actively participated in the writing of the manuscript. D.H. contributed to the statistical analysis and actively participated in the writing of the statistical sections of the manuscript. All authors critically reviewed the manuscript and approved the final version.

\section{References}

1. Torngren K, Ohman J, Salmi H, et al. Ticagrelor improves peripheral arterial function in patients with a previous acute coronary syndrome. Cardiology 2013;124:252-258.
2. Fromonot J, Dignat-Georges F, Rossi P, et al. Ticagrelor improves peripheral arterial function in acute coronary syndrome patients: relationship with adenosine plasma level. J Am Coll Cardiol 2016;67:1967-1968.

3. Xanthopoulou I, Vogiatzi C, Bampouri T, et al. Lack of evidence for deterioration in endothelial function following ticagrelor treatment cessation. Curr Vasc Pharmacol 2016;14:487-491.

4. Flammer AJ, Luscher TF. Human endothelial dysfunction: EDRFs. Pflugers Arch 2010;459:1005-1013.

5. Virdis A, Ghiadoni L, Taddei S. Human endothelial dysfunction: EDCFs. Pflugers Arch 2010;459:1015-1023.

6. Flammer AJ, Anderson T, Celermajer DS, et al. The assessment of endothelial function: from research into clinical practice. Circulation 2012;126:753-767.

7. Halcox JP, Schenke WH, Zalos G, et al. Prognostic value of coronary vascular endothelial dysfunction. Circulation 2002;106:653-658.

8. Bonetti PO, Pumper GM, Higano ST, et al. Noninvasive identification of patients with early coronary atherosclerosis by assessment of digital reactive hyperemia. J Am Coll Cardiol 2004;44:2137-2141.

9. Kuvin JT, Patel AR, Sliney KA, et al. Assessment of peripheral vascular endothelial function with finger arterial pulse wave amplitude. Am Heart J 2003;146:168-174.

10. Rubinshtein R, Kuvin JT, Soffler M, et al. Assessment of endothelial function by non-invasive peripheral arterial tonometry predicts late cardiovascular adverse events. Eur Heart J 2010;31:1142-1148.

11. Cattaneo M, Schulz R, Nylander S. Adenosine-mediated effects of ticagrelor: evidence and potential clinical relevance. J Am Coll Cardiol 2014;63:2503-2509.

12. Bonello L, Laine $M$, Kipson $N$, et al. Ticagrelor increases adenosine plasma concentration in patients with an acute coronary syndrome. J Am Coll Cardiol 2014;63:872-877.

13. Ohman J, Kudira R, Albinsson S, et al. Ticagrelor induces adenosine triphosphate release from human red blood cells. Biochem Biophys Res Commun 2012;418:754-758.

14. Grzesk G, Kozinski M, Navarese EP, et al. Ticagrelor, but not clopidogrel and prasugrel, prevents ADP-induced vascular smooth muscle cell contraction: a placebo-controlled study in rats. Thromb Res 2012;130:65-69.

15. Armstrong D, Summers $C$, Ewart $L$, et al. Characterization of the adenosine pharmacology of ticagrelor reveals therapeutically relevant inhibition of equilibrative nucleoside transporter 1. J Cardiovasc Pharmacol Ther 2014; 19:209-219.

16. Mehran R, Rao SV, Bhatt DL, et al. Standardized bleeding definitions for cardiovascular clinical trials: a consensus report from the Bleeding Academic Research Consortium. Circulation 2011;123:2736-2747.

17. Hamburg NM, Keyes MJ, Larson MG, et al. Cross-sectional relations of digital vascular function to cardiovascular risk factors in the Framingham Heart Study. Circulation 2008;1 17:2467-2474.

18. Sauder KA, West SG, McCrea CE, et al. Test-retest reliability of peripheral arterial tonometry in the metabolic syndrome. Diab Vasc Dis Res 2014;1 1:201-207.

19. Liu J, Wang J, Jin Y, et al. Variability of peripheral arterial tonometry in the measurement of endothelial function in healthy men. Clin Cardiol 2009;32:700-704.

20. McCrea CE, Skulas-Ray AC, Chow M, et al. Test-retest reliability of pulse amplitude tonometry measures of vascular endothelial function: implications for clinical trial design. Vasc Med 2012;17:29-36.

21. Selamet Tierney ES, Newburger JW, Gauvreau K, et al. Endothelial pulse amplitude testing: feasibility and reproducibility in adolescents. J Pediatr 2009;154:901-905.

22. Tomfohr LM, Martin TM, Miller GE. Symptoms of depression and impaired endothelial function in healthy adolescent women. J Behav Med 2008:31:137-143. 
23. Black MA, Cable NT, Thijssen DH, et al. Importance of measuring the time course of flow-mediated dilatation in humans. Hypertension 2008;51:203-210.

24. Harris RA, Padilla J, Hanlon KP, et al. Reproducibility of the flow-mediated dilation response to acute exercise in overweight men. Ultrasound Med Biol 2007;33:1579-1585.

25. Jarvisalo M, Jartti L, Marniemi J, et al. Determinants of short-term variation in arterial flow-mediated dilatation in healthy young men. Clin Sci (Lond) 2006;1 10:475-482.

26. Donald AE, Halcox JP, Charakida M, et al. Methodological approaches to optimize reproducibility and power in clinical studies of flow-mediated dilation. J Am Coll Cardiol 2008;51:1959-1964.

27. Ghiadoni L, Faita F, Salvetti $M$, et al. Assessment of flow-mediated dilation reproducibility: a nationwide multicenter study. J Hypertens 2012;30:1399-1405

28. Bailey DM, Rimoldi SF, Rexhaj E, et al. Oxidative-nitrosative stress and systemic vascular function in highlanders with and without exaggerated hypoxemia. Chest 2013;143:444-451.

29. Jayet PY, Rimoldi SF, Stuber T, et al. Pulmonary and systemic vascular dysfunction in young offspring of mothers with preeclampsia. Circulation 2010;122:488-494.

30. Rimoldi SF, Rexhaj E, Pratali L, et al. Systemic vascular dysfunction in patients with chronic mountain sickness. Chest 2012;141:139-146.

31. Rimoldi SF, Sartori C, Rexhaj E, et al. Antioxidants improve vascular function in children conceived by assisted reproductive technologies: a randomized double-blind placebo-controlled trial. Eur J Prev Cardiol 2015;22:1399-1407.

32. Iglarz M, Clozel M. Mechanisms of ET-1-induced endothelial dysfunction. J Cardiovasc Pharmacol 2007;50:621-628.
33. Sibal L, Agarwal SC, Home PD, et al. The role of asymmetric dimethylarginine (ADMA) in endothelial dysfunction and cardiovascular disease. Curr Cardiol Rev 2010;6:82-90.

34. Lu TM, Ding YA, Lin SJ, et al. Plasma levels of asymmetrical dimethylarginine and adverse cardiovascular events after percutaneous coronary intervention. Eur Heart J 2003;24:1912-1919.

35. Valkonen VP, Paiva H, Salonen JT, et al. Risk of acute coronary events and serum concentration of asymmetrical dimethylarginine. Lancet 2001;358:2127-2128.

36. Meinitzer A, Seelhorst U, Wellnitz B, et al. Asymmetrical dimethylarginine independently predicts total and cardiovascular mortality in individuals with angiographic coronary artery disease (the Ludwigshafen Risk and Cardiovascular Health study). Clin Chem 2007; 53:273-283.

37. Lip GY, Blann A. von Willebrand factor: a marker of endothelial dysfunction in vascular disorders? Cardiovasc Res 1997;34:255-265.

38. Zhukov O, Popov J, Ramos R, et al. Measurement of von Willebrand factor-FVIII binding activity in patients with suspected von Willebrand disease type 2N: application of an ELISA-based assay in a reference laboratory. Haemophilia 2009;15:788-796.

39. Castaman G, Tosetto A, Cappelletti A, et al. Validation of a rapid test (VWF-LIA) for the quantitative determination of von Willebrand factor antigen in type 1 von Willebrand disease diagnosis within the European multicenter study MCMDM-1VWD. Thromb Res 2010;126:227-231.

40. Vinholt PJ, Overgaard M, Diederichsen AC, et al. An ELISA for the quantitation of von Willebrand factor: osteoprotegerin complexes in plasma. Thromb Res 2013;131:396-400.

41. Hochberg Y, Benjamini Y. More powerful procedures for multiple significance testing. Stat Med 1990;9:811-818. 